\title{
Developing the XEOL and TR-XEOL at the X-ray Nanoprobe at Taiwan Photon Source
}

$\underline{\text { Bi-Hsuan Lin }}^{1,}{ }^{*}$, Shao-Chin Tseng ${ }^{1}$, Xiao-Yun Li ${ }^{1}$, Dai-Jie Lin ${ }^{2}$, Hsu-Cheng $\mathrm{Hsu}^{2}$, Yen-Ting Li ${ }^{1,3}, \mathrm{Yu}^{-}$ Cheng Chiu $^{3}$, Chien-Yu Lee ${ }^{1}$, Bo-Yi Chen ${ }^{1}$, Gung-Chian Yin ${ }^{1}$, Ming-Ying Hsu ${ }^{1}$, Shih-Hung Chang ${ }^{1}$, Wen-Feng Hsieh ${ }^{4}$ and Mau-Tsu Tang ${ }^{1}$

${ }^{1 .}$ National Synchrotron Radiation Research Center, Hsinchu 30076, Taiwan.

2. Department of Photonics, National Cheng Kung University, 701 Tainan, Taiwan.

${ }^{3 .}$ Department of Chemical Engineering, National Taiwan University of Science and Technology, Taipei 10607, Taiwan

4. Department of Photonics and Institute of Electro-Optical Engineering, National Chiao Tung University, Hsinchu 30010, Taiwan.

* Corresponding author, email_bihsuan@nsrrc.org.tw

The hard X-ray excited optical luminescence (XEOL) and time-resolved X-ray excited optical luminescence (TR-XEOL) have been developed successfully at the X-ray Nanoprobe (XNP) facility at Taiwan Photon Source (TPS). Not only the XNP at TPS provides $40 \mathrm{~nm}$ spatial resolution, but also the $\mathrm{X}$-ray energy of synchrotron source is continuous and tunable, which make the XEOL and TR-XEOL are powerful tool to study the optical properties and dynamic luminescence of novel optoelectronic materials.

TR-XEOL spectra were collected by a fiber, which is coupled to a spectrometer (iHR320, HORIBA) with Hamamatsu C10910 streak camera and M10913 slow single sweep unit. The repetition rate and the temporal resolution of the single bunch mode at TPS are about $578 \mathrm{KHz}$ and $30 \mathrm{ps} 1.72 \mu \mathrm{s}$, respectively. Comparing other time resolved methods, the advantage of using streak camera based TRXEOL is the ability to simultaneously obtain both emission wavelength data and lifetime decay [1]. Figure 1 shows the abilities to study the optical and dynamic properties of the trihalide perovskite $\mathrm{CH}_{3} \mathrm{NH}_{3} \mathrm{PbBr}_{3}$ by using XEOL and TR-XEOL. From the XEOL measurements, the emission wavelength was located at about $535 \mathrm{~nm}$, which is shown in Fig. 1(a). Then, the streak image with 500 ns sweep time, and the lifetime decay can be obtained by using TR-XEOL, which are shown in Fig. 1(b) and $1(\mathrm{c})$, respectively. The fitting results of the lifetime decay were $\tau_{1}=6.4 \mathrm{~ns}$ and $\tau_{2}=64.9 \mathrm{~ns}$.

In our previous reports using unfocused X-ray beam at Taiwan Light Source (TLS), we have demonstrated not only the temperature-dependent XEOL to study the optical properties of $\mathrm{O}$ and $\mathrm{Zn}$ polarity of a $c$-plane $\mathrm{ZnO}$ wafer [2], but also the polarization-dependent XEOL to study the crystallographic orientations of a non-polar $a$-plane $\mathrm{ZnO}$ wafer [3]. In this study [4], we used XEOL with nano-focused X-ray beam to study the optical properties of $\mathrm{ZnO}$ microrod. Figure 2(a) shows the SEM image of the $\mathrm{ZnO}$ microrod on Ga-polar $c$-plane $\mathrm{GaN}$ substrate. The diameter of $\mathrm{ZnO}$ microrod is about $5 \mu \mathrm{m}$ with a hexagonal shape. Base on the benefit of nano-focused beam, the optical properties can be analyzed from specific nano-position of the $\mathrm{ZnO}$ microrod as shown in Fig. 2(b)-(e). The nearband-edges (NBE) of $\mathrm{ZnO}$ and $\mathrm{GaN}$ were measured at $3.299 \mathrm{eV}$ and $3.369 \mathrm{eV}$, respectively. Besides the XEOL spectra, the XEOL mapping as well as the X-ray fluorescence (XRF) mapping was also developed at XNP. SEM, XRF mapping, XEOL mapping of GaN NBE, and XEOL mapping of ZnO NBE are shown in Fig. 3(a)-(d), respectively. From the XEOL mapping, we can obtain the information of the emission distribution of $\mathrm{GaN}$ and $\mathrm{ZnO} \mathrm{NBE}$. Then, the $\mathrm{Zn}$ element distribution can be measured 
by XRF mapping. After accomplished these test experiments, we believe that the XNP has the abilities and opportunities to investigate and explore the novel optoelectronic materials and nanotechnology.

\section{References:}

[1] M. J. Ward, T. Z. Regier, J. M. Vogt, R. A. Gordon, W. Q. Han, and T. K. Sham. Time-resolved Xray excited optical luminescence using an optical streak camera. in Journal of Physics: Conference Series. 2013. IOP Publishing.

[2] Bi-Hsuan Lin, Huang-Yeh Chen, Shao-Chin Tseng, Jian-Xing Wu, Bo-Yi Chen, Chien-Yu Lee, Gung-Chian Yin, Shih-Hung Chang, Mau-Tsu Tang, and Wen-Feng Hsieh, Applied Physics Letters 109, 192104 (2016).

[3] Bi-Hsuan Lin, Yung-Chi Wu, Huang-Yeh Chen, Shao-Chin Tseng, Jian-Xing Wu, Xiao-Yun Li, BoYi Chen, Chien-Yu Lee, Gung-Chian Yin, and Shih-Hung Chang, Optics express 26, 2731-2739 (2018).

[4] Ministry of Science and Technology of Taiwan (MOST 106-2112-M-213-005) and the National Synchrotron Radiation Research Center provided support for this research.
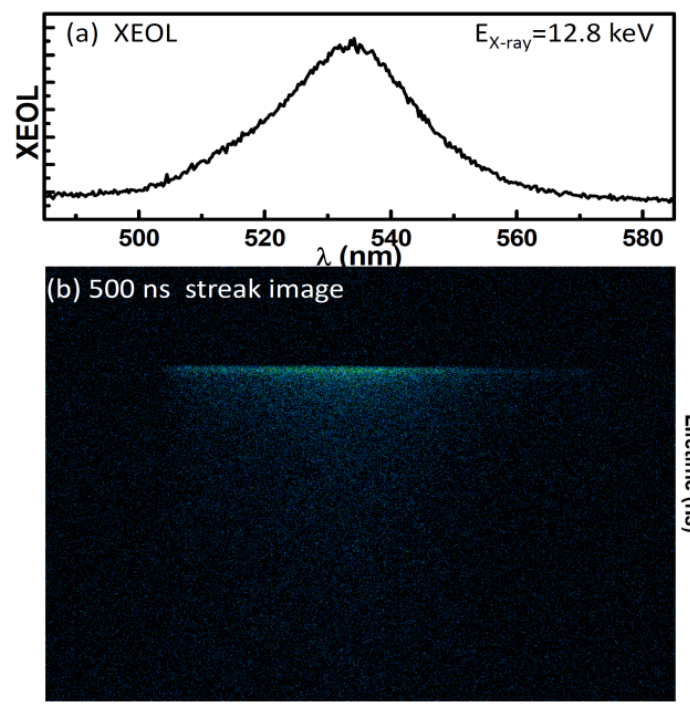

Figure. 1. (a) XEOL spectrum, (b) Streak image, and (c) TR-XEOL spectrum.

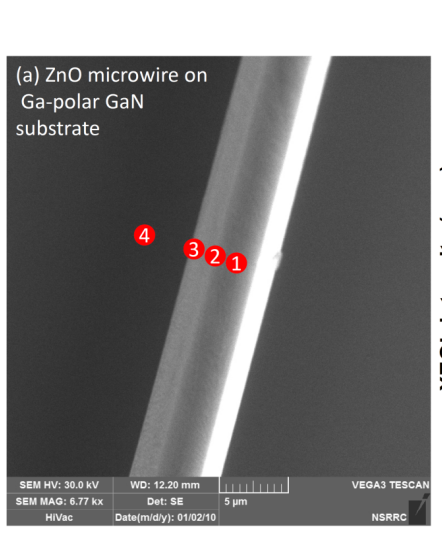

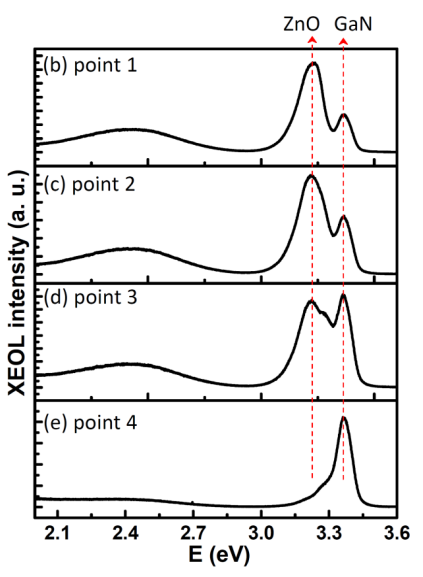
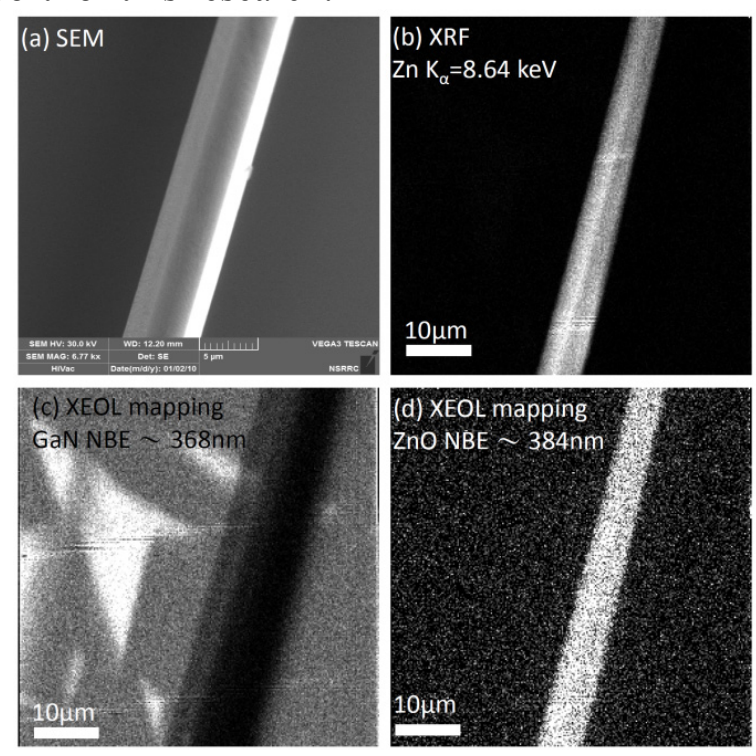

Figure. 3. (a) SEM image, (b) XRF mapping, (c)XEOL mapping of GaN NBE, and (d) XEOL mapping of $\mathrm{ZnO} \mathrm{NBE}$ of $\mathrm{ZnO}$ microrod.

Figure. 2. (a) SEM image of $\mathrm{ZnO}$ microrod, and (b)-(e) XEOL spectra. 